\title{
New Full-Range Electron Tomography Procedure for Accurate Quantification of Surfaces, Curvature, and Porosity in Energy-Related Nanomaterials
}

\author{
Elliot Padgett ${ }^{1}$, Robert Hovden ${ }^{1}$, Jessica A. C. Da Silva ${ }^{2}$, Tobias Hanrath ${ }^{2}$, and David A. Muller ${ }^{1}$. \\ 1. School of Applied and Engineering Physics, Cornell University, Ithaca, NY. \\ 2. School of Chemical and Biomolecular Engineering, Cornell University, Ithaca, NY.
}

Accurate quantitative analysis of 3D nanostructures is essential for establishing structure-property relationships in many energy materials, especially catalysts and their supports. However, obtaining quantitative results from conventional electron tomography is hindered by the missing wedge from a limited tilt range, which causes distortions in the geometry and intensities of reconstructed tomograms, including false porosity near high-Z catalysts. Full-rotation tomography using an on-axis tomography holder can eliminate the missing wedge and resolve these problems. Most such work has been accomplished using focused ion beam (FIB) milling of needle-shaped specimens [1]. This is suitable for bulk or device specimens where local lift-out is a necessary part of TEM sample preparation, but it is cumbersome for many nanostructured energy materials such as catalyst nanoparticles because FIB preparation is slow, expensive, potentially damaging, and may require embedding. Here we present a new approach to full-rotation tomography sample preparation using carbon nanofibers (CNF) as a 1D specimen support and show the quantitative analyses now enabled by the high-fidelity tomograms.

In a simple, bench-top procedure, carbon fibers are collected on a tungsten needle, and sample particles are then collected on the fibers (Figure 1a,b). This method could be applied to study nanoparticle and microparticle materials used as catalysts and supports for fuel cells, water splitting, and fuel processing, or in electrodes for batteries and supercapacitors. Here, we demonstrate this technique with golddecorated cubic strontium titanate particles (Figure 1b), used as photocatalysts for water splitting. It is also suitable for some nanofiber-based materials, such as CNF-supported Pt fuel cell catalysts [2], as shown in Figure 1c,d. Tomographic reconstructions of each sample, produced using the simple, well understood weighted back-projection algorithm, are shown in Figure 1b-d.

Eliminating the missing wedge provides several benefits for quantitative analysis of structural properties relevant to energy materials performance. Missing wedge artifacts introduce blurring and shadow artifacts, resulting in problems for segmentation including distorted geometry and false pores, as shown for a $\pm 75^{\circ}$ tilt range in Figure $2 \mathrm{a}$. In contrast, the full rotation tomogram cross-sections in Figure $1 \mathrm{~d}$ and Figure 2a have no artifacts due to the missing wedge, and show clear, undistorted geometry and no spurious intensity variations. Consequently, intensities in the tomogram quantitatively reflect the scattering density of materials in the specimen and different materials show clear peaks in the tomogram intensity histogram (Figure 2a). The full rotation Au/STO tomogram can be accurately segmented with straightforward, automatic threshold-based methods to separate Au, STO, and pores (Figure 2b). The full rotation tomogram also permits analysis of surface structure properties such as curvature (Figure 2c) and faceting, which are distorted in conventional electron tomography. Full rotation tomography with CNF supports thus allows accurate determination of nanoparticle pore size and connectivity, and surface area and curvature, key parameters for electrochemical energy materials [3].

References: 
[1] N Kawase, et al, Ultramicroscopy 107 (2007), 8-15.

[2] BDA Levin et al, Scientific Data 3, (2016), 160041.

[3] E Padgett acknowledges support from a NSF Graduate Research Fellowship (DGE-1650441), DOE

(DE-SC0011385), \& Electron microscopy facility support from NSF MRSEC program (DMR 1120296).

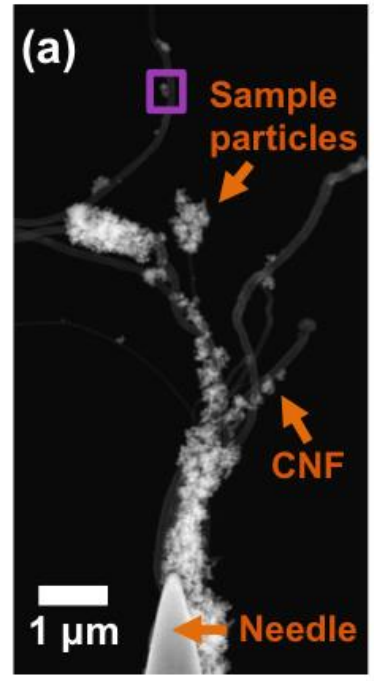

(b)

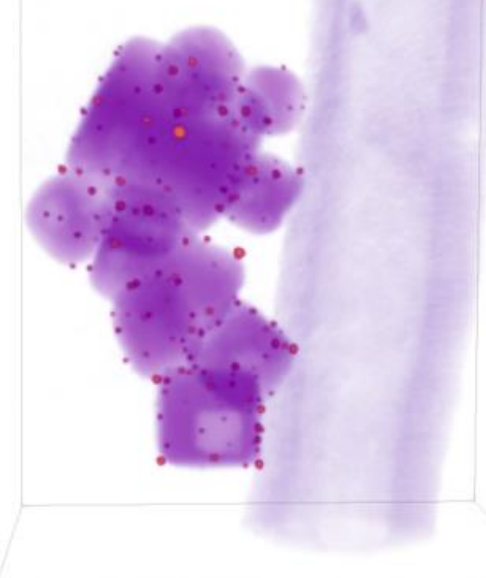

(c)

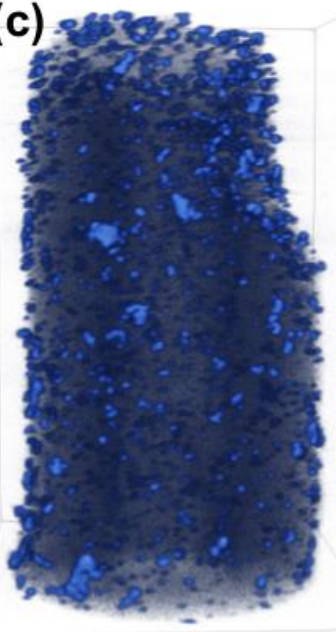

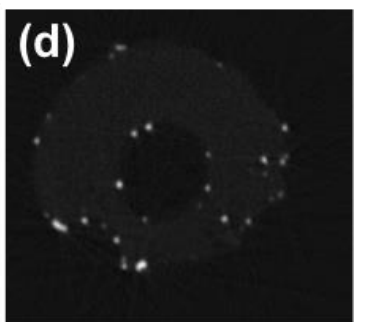

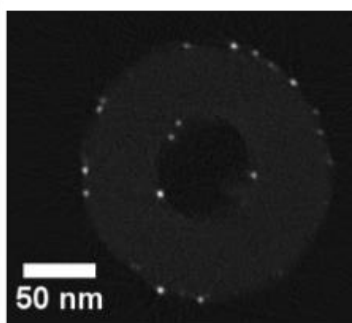

Figure 1. (a) STEM image of Au/STO showing needle, support fibers, and sample nanoparticles. 3D volume renderings of tomograms for Au/STO (b) and Pt/CNF (c). (d) Cross-sections of Pt/CNF tomograms perpendicular to tilt axis, showing absence of missing wedge or other artifacts.
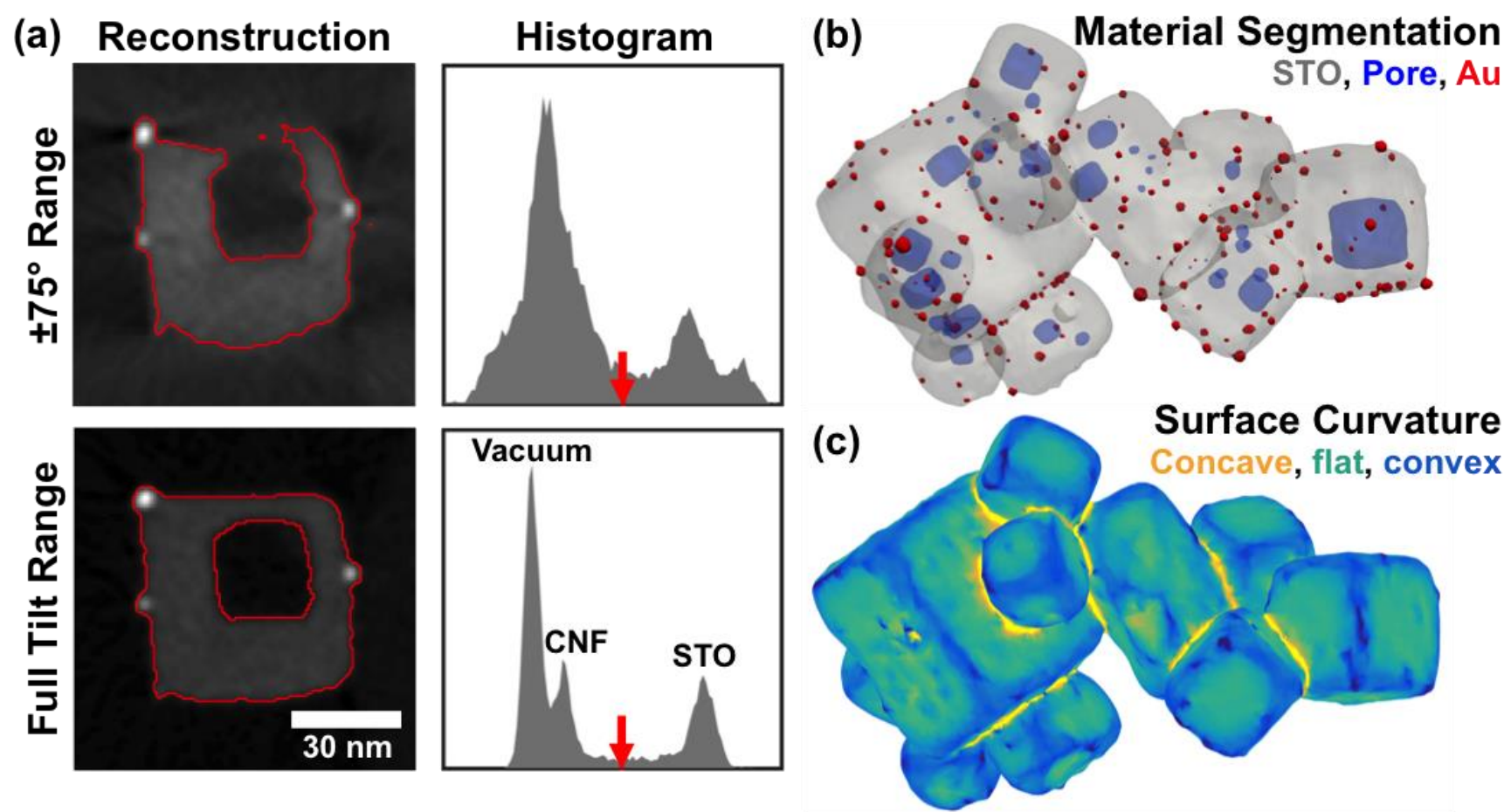

Figure 2. (a) Comparison of tomogram cross-sections and intensity histograms for $\pm 75^{\circ}$ tilt range and full tilt range. Red arrows and lines indicate segmentation threshold chosen by Otsu's method. (b) 3D surface rendering of tomogram segmented by threshold and morphological filtering, showing $\mathrm{Au}$ particles, STO, and internal pores. (c) 3D rendering of STO surface with color indicating local mean curvature, separating curved convex (blue) and concave (yellow) regions from flat facets (green). 